Article

\title{
Electronic Origin of the Orthorhombic Cmca Structure in Compressed Elements and Binary Alloys
}

\author{
Valentina F. Degtyareva \\ Institute of Solid State Physics RAS, Moscow district, Chernogolovka 142432, Russia; \\ E-Mail: degtyar@issp.ac.ru; Tel.: +7-496-522-8376; Fax: +7-496-522-8160
}

Received: 24 May 2013; in revised form: 21 June 2013 / Accepted: 15 July 2013 /

Published: 19 July 2013

\begin{abstract}
Formation of the complex structure with 16 atoms in the orthorhombic cell, space group Cmca (Pearson symbol $o C 16$ ), was experimentally found under high pressure in the alkali elements ( $\mathrm{K}, \mathrm{Rb}, \mathrm{Cs}$ ) and polyvalent elements of groups IV ( $\mathrm{Si}, \mathrm{Ge}$ ) and V (Bi). Intermetallic phases with this structure form under pressure in binary Bi-based alloys (Bi-Sn, Bi-In, Bi-Pb). Stability of the Cmca-oC16 structure is analyzed within the nearly free-electron model in the frame of Fermi sphere-Brillouin zone interaction. A Brillouin-Jones zone formed by a group of strong diffraction reflections close to the Fermi sphere is the reason for the reduction of crystal energy and stabilization of the structure. This zone corresponds well to the four valence electrons in $\mathrm{Si}$ and $\mathrm{Ge}$, and leads to assume an spd-hybridization for Bi. To explain the stabilization of this structure within the same model in alkali metals, that are monovalents at ambient conditions, a possibility of an overlap of the core, and valence band electrons at strong compression, is considered. The assumption of the increase in the number of valence electrons helps to understand sequences of complex structures in compressed alkali elements and unusual changes in their physical properties, such as electrical resistance and superconductivity.
\end{abstract}

Keywords: crystal structure; Hume-Rothery phases; structure stability

\section{Introduction}

Experimental studies of elements under high pressure reveal a great variety of structural transformations, summarized in review papers (see [1-3] and literature cited therein). At ambient pressure, the most representative structures for elements are close-packed structures: body-centred 
cubic (bcc), face-centred cubic (fcc), and hexagonal close-packed (hcp), typical for metals. Elements on the right-hand side of the Periodic table - main group IV-VII semi-metals and non-metals - crystallize in open-packed structures following the $8-\mathrm{N}$ coordination rule. These elements tend to transform under pressure to a metallic state with closely packed structures. On the left-hand side of the Periodic table, the group 1 and II elements (the alkali and alkali-earth elements) are good metals at ambient pressure that crystallize in the closely packed structures. Under pressure, however, they show an anomalous behavior, transforming to open-packed structures.

Two different trends from both sides of the Periodic table are illustrated by the data in Table 1, which show the structural sequences for $\mathrm{Si}, \mathrm{Ge}$, and $\mathrm{Bi}$ (upper panel), and for the heavy alkali elements (lower panel). These elements are chosen for our consideration because, under pressure, they all go in their pathways through the same structure-orthorhombic with 16 atoms in the cell, space group Cmca, Pearson symbol $o C 16$. This type of structure is unknown for any element or compound at ambient pressure, and was first defined for Cs and Si under pressure [4,5], and then for other elements included in Table 1. Theoretical calculations of the band structure energy for Cmca phases of Si and Cs have demonstrated that "structural similarities in Cs-V and Si-VI are not reflected in similarities of the electronic structures" [6]. For Si, a nearly free-electron behavior has been shown, while for Cs-V, dominate $d$-orbital components due to $s$ - $d$ electron transfer have been found [6].

Table 1. Sequences of structural transformations on pressure increase for elements with the $o C 16$ structure.

Element

Structure sequences

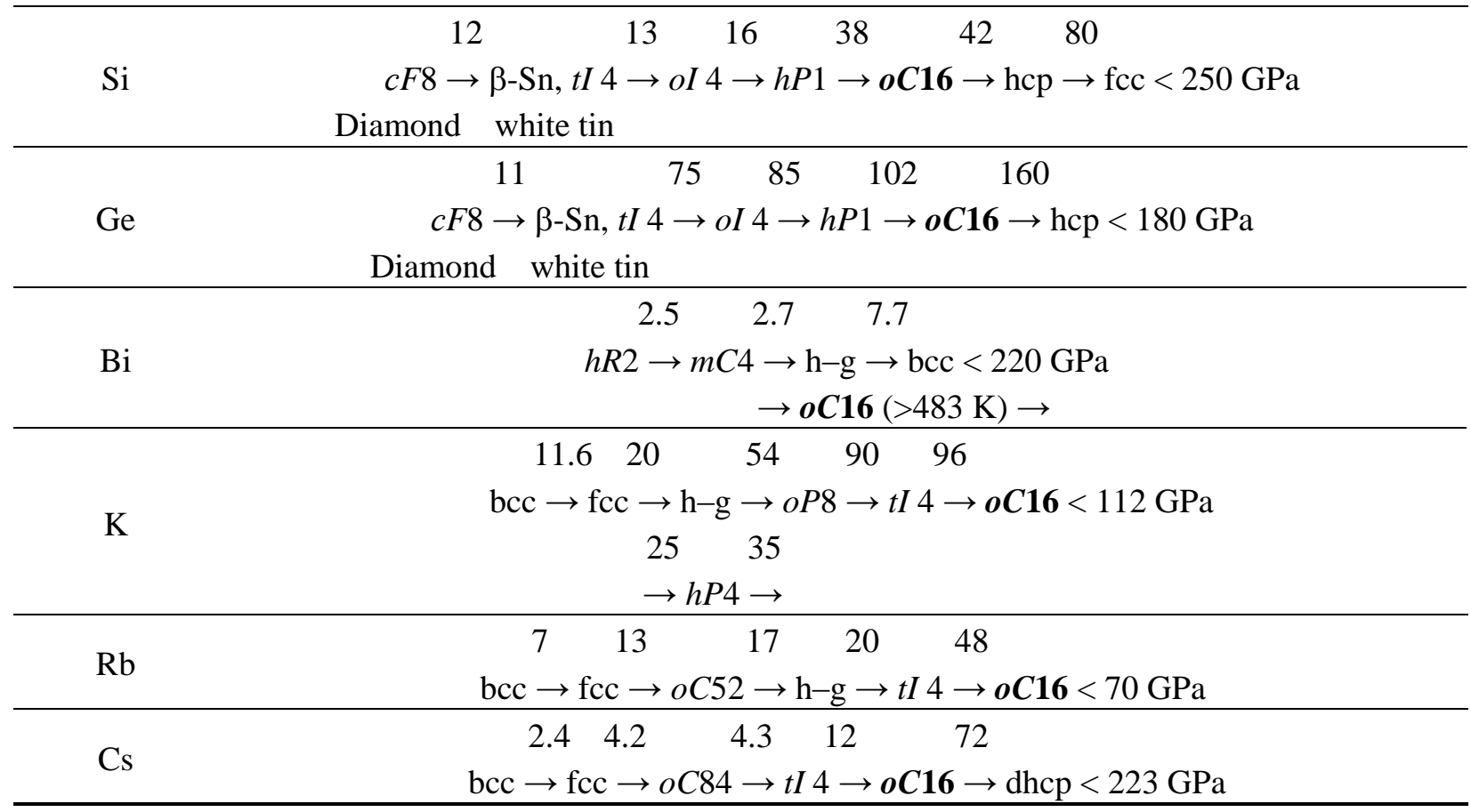

Notes: The numbers above the arrows show the transition pressures in GPa, reported in experimental measurements on pressure increase. The crystal structures of the phases are denoted with their Pearson symbols, apart from the most common metallic structures bcc, fcc, and hcp. "h-g" stands for a host-guest structure. For experimental data see [1-3] and literature cited therein. 
In this paper, formation and stability of the $o C 16-\mathrm{Cmca}$ structure is discussed within the Brillouin zone-Fermi sphere interaction with application of the Hume-Rothery mechanism [7-9]. A complex structure, like $\gamma$-brass $\mathrm{Cu}_{5} \mathrm{Zn}_{8}$-cI52, reduces its crystal energy by formation of the Brillouin zone planes close to the Fermi sphere, satisfying the electron concentration rule. Following this model it is necessary to assume for compressed alkali metal, not only $s$ - $d$ transfer, but also further overlap of valence band and upper core electrons [10]. This assumption was made to account for an unusual behavior under pressure of the alkali element sodium [11]. Na transforms above 1 Mbar to the low-symmetry, open structure $o P 8$, which is similar to the phase AuGa with two electrons per atom. It was suggested for $\mathrm{Na}-o P 8$, an electronic transfer from core into the valence band. A similar suggestion is necessary to assume for alkali elements in $o C 16-C m c a$ phase, as is discussed in this paper.

\section{Results and Discussion}

The crystal structure $o C 16-\mathrm{Cmca}$ belongs to the family of structures described by Pearson [12] as a planar square-triangle nets of atoms, assigned as $3^{2} 434$ where " 3 " specifies a triangle, "4" a square, and the superscript shows a multiplicity. These nets are alternated with square $4^{4}$ nets. Layers $3^{2} 434$ may be stacked either antisymmetric or with a shift on $1 / 2$ of a basic axis. Examples of this family represent the compounds $\mathrm{CuAl}_{2}(t I 12), \mathrm{CoGe}_{2}$ (oC24), $\mathrm{PdSn}_{2}$ (tI48), $\mathrm{PdSn}_{3}$ (oC32), and others [12]. An interesting example is an $o C 20$ phase found for $\mathrm{PdSn}_{4}, \mathrm{PtSn}_{4}$, and $\mathrm{AuSn}_{4}$ [13]. This type of structure, with the space group Aba2, has some similarities with the discussed $o C 16$-Cmca structure having nearly same axial ratios but slightly different stacking layers: four $3^{2} 434$ nets alternate by two $4^{4}$ nets, whereas in $o C 16-C m c a$ the numbers of nets are two and four, respectively, as considered below.

\subsection{Structural Characteristics of oC16-Cmca Phases}

The conventional unit cell contains 16 atoms in two crystallographically non-equivalent positions $8 \mathrm{f}(0, y, z)$ and $8 \mathrm{~d}(x, 0,0)$ in Wyckoff notation of the Cmca space group. A feature of the structure $o C 16$ - Cmca is $3^{2} 434$ nets, formed by atoms in the 8 f position, with squares lying over the cell corners and cell base centers, or over the midpoints of the cell edges, and alternated by two square nets $4^{4}$ (atoms in the $8 \mathrm{~d}$ position) shifted with respect to each other on a half of the $b$ axis as shown in Figure 1. The configuration of atoms in the $3^{2} 434$ nets implies a rigid atomic arrangement with atomic positions $y$ and $z$ (8f) close to 0.167 and 0.333 , respectively, providing $y+z \approx 0.5$.

The data of all oC16-Cmca phases found at present are summarized in Table 2. All the structures have very similar axial ratios $(b \approx c \approx 1, a / b \approx a / c \approx \sqrt{ } 3)$ and very similar atomic positions. This indicates that the structure is defined by certain criteria related to the electronic structure.

Some data for Cmca-oC16 phases in the Table 2 are essentially different, namely volume compression, $\mathrm{V} / \mathrm{V}_{\mathrm{o}}$, which is especially significant for alkali metals (indicated in bold). The shortest interatomic distances for alkali metals $\mathrm{Cs}, \mathrm{Rb}$, and $\mathrm{K}$ (see Table 2, in bold) correspond to atomic radii $1.62,1.38$, and $1.16 \AA$, respectively. It is interesting to compare these values to the ionic radii given by Kittel [14], 1.67, 1.48, and $1.38 \AA$, or to the ionic radii for coordination number 10 given by Shannon [15], 1.81, 1.66, and $1.59 \AA$, respectively. At this degree of compression it is necessary to consider ionic core overleap and a transfer of core electrons into the valence band, as was theoretically proposed earlier [10]. 
Figure 1. Crystal structure $o C 16-\mathrm{Cmca}$ of Si-VI. (a) A perspective view. The atomic positions $8 \mathrm{f}$ and $8 \mathrm{~d}$ are denoted as red and yellow, respectively; (b) A projection along the $a$-axis for nets at $x=0.5$ (red) and $x=0.218$ and 0.282 (yellow). The structure can be viewed as an alternating sequence of planar layers formed by square-triangle nets and puckered nearly square layers. The shortest interatomic distances are marked by dark lines (modified from [4]).

(a)

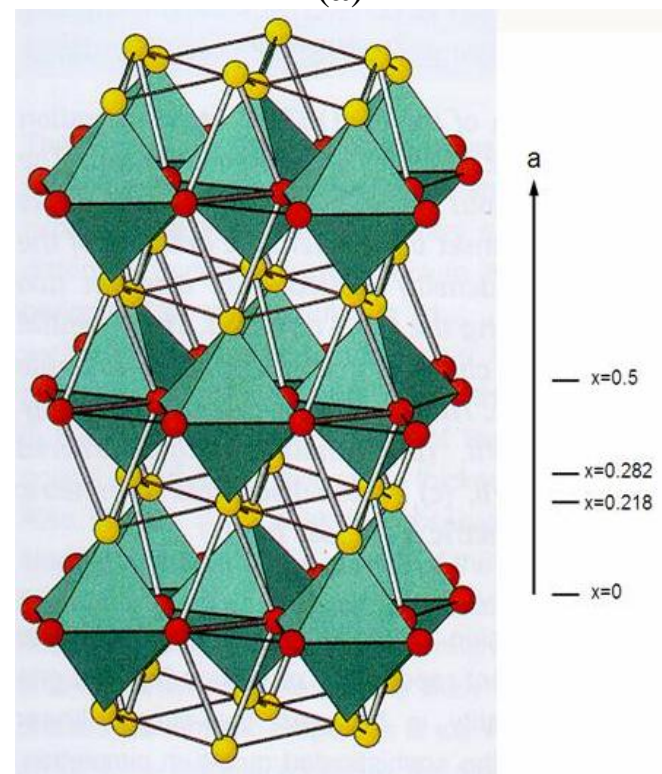

(b)

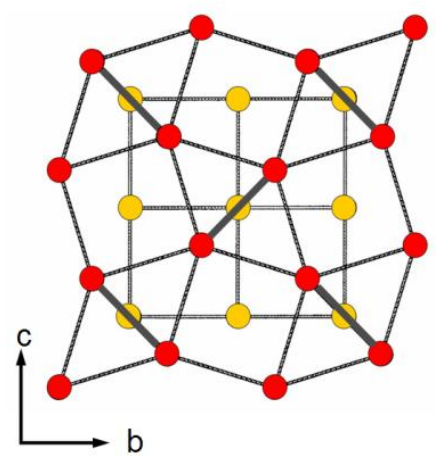

Table 2. Structural details of all the known $\mathrm{Cmca-oC16}$ phases, formed under pressure in the pure elements $\mathrm{Cs}, \mathrm{Rb}, \mathrm{Si}, \mathrm{Ge}$, and $\mathrm{Bi}$, and in Bi-alloys with $\mathrm{In}, \mathrm{Sn}$, and $\mathrm{Pb}$ (experimental data are taken from references indicated for each material).

\begin{tabular}{|c|c|c|c|c|c|c|c|c|c|}
\hline Phase & $\begin{array}{c}\text { Si-VI } \\
\text { 38.4 GPa } \\
{[5]} \\
\end{array}$ & $\begin{array}{c}\text { Ge } \\
135 \text { GPa } \\
{[16]} \\
\end{array}$ & $\begin{array}{c}\text { Cs-V } \\
12 \text { GPa [4] }\end{array}$ & $\begin{array}{c}\text { Rb-VI } \\
48.1 \mathrm{GPa} \\
{[17]} \\
\end{array}$ & $\begin{array}{c}\text { K-VI } \\
112 \text { GPa } \\
{[18]} \\
\end{array}$ & $\begin{array}{c}\text { Bi-IV } \\
\text { 3.2 GPa } \\
\text { 465 K [19] }\end{array}$ & $\begin{array}{c}\mathrm{Bi}_{40} \mathrm{Sn}_{60} \\
5.8 \mathrm{GPa}[20]\end{array}$ & $\begin{array}{c}\mathrm{Bi}_{80} \mathrm{~Pb}_{20} \\
\text { quenched from } \\
1.7 \mathrm{GPa}[21]\end{array}$ & $\begin{array}{c}\mathrm{Bi}_{70} \mathbf{I n}_{30} \\
\text { quenched from } \\
2.0 \mathrm{GPa}[21] \\
\end{array}$ \\
\hline$a(\AA)$ & 8.024 & 7.885 & 11.205 & 9.372 & 8.032 & 11.191 & 10.7345 & 11.209 & 11.236 \\
\hline$b(\AA)$ & 4.796 & 4.655 & 6.626 & 5.5501 & 4.753 & 6.622 & 6.3270 & 6.672 & 6.555 \\
\hline$c(\AA)$ & 4.776 & 4.651 & 6.595 & 5.5278 & 4.716 & 6.608 & 6.3115 & 6.658 & 6.531 \\
\hline $\mathrm{V}_{\mathrm{at}}\left(\AA^{3}\right)$ & 11.49 & 10.67 & 30.60 & 17.97 & 11.25 & 30.61 & 26.79 & 31.12 & 30.07 \\
\hline $\mathrm{V} / \mathrm{V}_{\mathrm{o}}$ & 0.565 & 0.470 & 0.264 & 0.194 & 0.150 & 0.864 & 0.882 & 0.905 & 0.921 \\
\hline$a / c$ & 1.680 & 1.695 & 1.699 & 1.689 & 1.703 & 1.693 & 1.701 & 1.684 & 1.721 \\
\hline$b / c$ & 1.004 & 1.001 & 1.005 & 1.004 & 1.004 & 1.002 & 1.002 & 1.002 & 1.004 \\
\hline $\mathrm{d}_{\text {short }}(\AA)$ & 2.340 & 2.315 & 3.235 & 2.764 & 2.317 & 3.275 & 2.985 & 3.242 & 3.146 \\
\hline y (8f) & 0.173 & 0.173 & 0.164 & 0.170 & 0.173 & 0.170 & 0.167 & 0.172 & 0.170 \\
\hline$z(8 f)$ & 0.327 & 0.328 & 0.313 & 0.318 & 0.327 & 0.320 & 0.333 & 0.328 & 0.330 \\
\hline $\mathrm{x}(8 \mathrm{~d})$ & 0.216 & 0.218 & 0.218 & 0.211 & 0.216 & 0.210 & 0.212 & 0.214 & 0.212 \\
\hline
\end{tabular}

\subsection{Electronic Origin of Stability for oC16 Phases: Hume-Rothery Effects}

Examination of stability of the $o C 16$ structure is based on the concept of the Hume-Rothery mechanism and consists of construction of the Brillouin planes close to the Fermi sphere defined 
within the nearly free-electron model [22]. We first consider the $o C 16$ phase in $s p$-elements and alloys and then in alkali elements.

\subsubsection{Group IV Elements Si and Ge with the $o C 16$ Structure}

Elements $\mathrm{Si}$ and $\mathrm{Ge}$ are $s p$-elements of group IV and therefore have four valence electrons per atom. Figure $2 \mathrm{a}$ shows diffraction pattern for $\mathrm{Si}-o C 16$ with indication of position $2 \mathrm{k}_{\mathrm{F}}$ for $z=4$ that locates just above a group of strong diffraction peaks selected for construction of the Brillouin-Jones zone (left) [22]. The inscribed Fermi sphere corresponds to $2 \mathrm{k}_{\mathrm{F}}$ for $z=4$ and is in contact with 36 planes; zone filling by electron states $\mathrm{V}_{\mathrm{FS}} / \mathrm{V}_{\mathrm{BZ}}$ equals $\sim 93 \%$ as for the classical Hume-Rothery phase $\gamma$-brass $\mathrm{Cu}_{5} \mathrm{Zn}_{8}$. For the spherical Fermi surface, calculated distances to the Bragg planes (131), (113), (421), and (511) are slightly below the value of $\mathrm{k}_{\mathrm{F}}$ with ratios 1.032, 1.028, 1.014, and 1.004, respectively. In a real case, there is a degree of deformation of the Fermi sphere by the contact with the Brillouin boundary, so-called "truncation" factor. This was initially suggested by Sato and Toth for the electronic nature of long-period superlattices in alloys like $\mathrm{Cu}-\mathrm{Au}$, and similar systems [23], where the value of this factor was found to be within $5 \%$.

In the middle of Figure 2a a Brillouin zone is shown, which is constructed by the planes of the first group of the strong reflections lying within the Fermi sphere. It is remarkable, that due to particular ratios of cell axes, this polyhedron is very symmetrical with hexagonal cross-sections $\perp c^{*}$ and in planes $a^{*} c^{*}$ and $b^{*} c^{*}$. The symmetry of the BZ polyhedron formed by first strong Bragg planes is reflected on the electrostatic energy (see by Harrison [24]).

\subsubsection{Structure $o C 16$ in $\mathrm{Bi}$ and Bi-alloys}

Crystal structure of high-pressure, high-temperature phase of bismuth at $\sim 4 \mathrm{GPa}$ and $\sim 500 \mathrm{~K}$ was recently solved by single-crystal synchrotron X-ray diffraction [19], with characteristics given in Table 2. Experimental observations of this $\mathrm{Bi}$ phase have a long story, since 1958 [25]. Pressure-temperature ranges for this $\mathrm{Bi}$ phase, assigned now as $\mathrm{Bi}-\mathrm{IV}$, are from $\sim 2.2 \mathrm{GPa}$ to $\sim 5.5 \mathrm{GPa}$ above $450 \mathrm{~K}$. Room temperature phase $\mathrm{Bi}$-III, stable at pressures $2.7-7.7 \mathrm{GPa}$, has a complex incommensurate host-guest structure [26]. Phase boundary Bi-III-Bi-IV is nearly horizontal $(\sim 450 \mathrm{~K})$ and both phases transform to $\mathrm{Bi}-\mathrm{V}$ with a body-centered cubic structure at higher pressure. There is a small volume difference between Bi phases III and IV, and different structures may be expected from the difference in valence electron bands. Formally, the group V element Bi has outer $s^{2} p^{3}$ electron configuration. Under pressure-temperature conditions, one may expect that hybridization of $s, p$, and $d$ levels occur due to lowering of the higher empty $d$-band, as discussed by Pearson (see Reference [12], pp. 230-232).

For stability of the $o C 16$ structure four valence electrons are necessary, and this count relates to the assumption of $s p d$ hybridization. It should be noted that for considerations of the Fermi sphere radius within the model of FS-BZ interactions only $s p$ electrons are taken into account as structure controlled electrons. The same assumption on $s p d$ hybridization for $\mathrm{Bi}$ in Bi-based alloys with the $o C 16$ structure is necessary to obtain the electron per atom count, equal to four, which stabilizes this structure.

Intermediate phases in $\mathrm{Bi}-\mathrm{Pb}$ and $\mathrm{Bi}$-In alloys were obtained under pressure-temperature conditions and quenched to ambient pressure at liquid nitrogen temperature [27]. Diffraction patterns of both 
phases, $\mathrm{Bi}_{80} \mathrm{~Pb}_{20}$ and $\mathrm{Bi}_{70} \mathrm{In}_{30}$, were similar, however, rather complex to be solved 40 years ago. Development of new experimental methods of X-ray diffraction under pressure, and new programs of structure solutions, allowed defining oC16-Cmca structure in $\mathrm{Si}$ and $\mathrm{Cs}$, and other elements, as indicated in Table 2. Based on these findings, a description of structure was found for similar phases in $\mathrm{Bi}_{80} \mathrm{~Pb}_{20}$ and $\mathrm{Bi}_{70} \mathrm{In}_{30}$ alloys [21], and was also suggested for the Bi-IV phase. Experimental confirmation of this suggestion was obtained recently [19].

In the Bi-Sn alloys, an intermediate high-pressure phase was observed in situ under pressure [20]. It is remarkable that the X-ray diffraction data of the $\mathrm{Bi}_{40} \mathrm{Sn}_{60}$ phase reveals the $o C 16$-Cmca structure with the evidence of site-ordering. Additional diffraction peaks (for example, the difference reflection 111) indicate preferred occupation of the constituent atoms, $\mathrm{Sn}$ and $\mathrm{Bi}$, in positions $8 \mathrm{f}$ and $8 \mathrm{~d}$, respectively. To extend this observation, one can expect formation of ordered oC16-Cmca structures in some binary systems under pressure on the condition that the electron concentration rule will be satisfied.

\subsubsection{Structure $o C 16$ in Alkali Elements: Core Ionization}

Phases with oC16-Cmca structure appear in $\mathrm{Si}$ and $\mathrm{Ge}$ under pressure within the sequence $c F 8 \rightarrow t I 4 \rightarrow h P 1 \rightarrow o C 16 \rightarrow h c p$, which follows an increase in packing density and coordination number $(\mathrm{CN}) 4 \rightarrow 6 \rightarrow 6+2 \rightarrow 10,11 \rightarrow 12$. This pathway is in general tendency, for the elements on the right-hand side of the Periodic table, to adopt the more close-packed structures. The opposite trend was found for the elements on the left-hand side - a transformation under compression to open-packed structures. Alkali elements with the bcc structure at ambient conditions, which transforms to the fcc on pressure increase ( $\mathrm{CN}=8$ and 12, respectively), undergo a transformation to low-coordinated structures. For $\mathrm{K}, \mathrm{Rb}$, and $\mathrm{Cs}$ the structure before $o C 16$ is $t I 4$ with $\mathrm{CN}=(4+4)$, so that there is a turnover at transition $t \mathrm{I} 4 \rightarrow o C 16$ where $\mathrm{CN}$ starts to increase again, and for $\mathrm{Cs}$ it attains $\mathrm{CN}=12$ at the transition to the double hexagonal close-packed structure, dhcp.

Unexpected behavior of alkali elements on compression tolerates radical changes in valence electron bonding, and an electronic level overlap and hybridization of upper core electrons and valence band should be considered. This assumption is supported by the large compression and short interatomic distances in $o C 16$ structure, as is analyzed above, in Section 2.1. On Figure $2 \mathrm{~b}$ diffraction pattern Cs-oC16 is shown (left) with the calculated $2 \mathrm{k}_{\mathrm{F}}$ positions shown for two possible numbers of valence electrons, $z=1$ and 4 . The groups of strong reflections near these positions are used for BZ construction (middle and right). The relation of the FS and BZ for the case $z=1$ does not satisfy the Hume-Rothery conditions that supports the suggestion in increase of the valence electron counts. The Brillouin zone in Figure 2b (right) for $z=4$ is similar to that of Si-oC16 in Figure 2a.

It is remarkable that $o C 16$ structure is found in heavy alkalis, $\mathrm{K}, \mathrm{Rb}$, and $\mathrm{Cs}$, but not in light, $\mathrm{Na}$ and Li. For $\mathrm{Na}$, transformations from fcc to complex phases started at pressures above $100 \mathrm{GPa}$, whereas for $\mathrm{K}, \mathrm{Rb}$, and $\mathrm{Cs}$, at 20, 13, and $4.3 \mathrm{GPa}$, respectively (see references [1-3]). This different behavior is related to the energetics of the electronic levels. First of all it should be noted that position of the empty $d$-level for $\mathrm{Na}$ is well above the valence $s$-level comparing to $s$ - $d$ levels for heavy alkalis. The $o C 16$ structure may be expected for $\mathrm{Na}$ at higher compression, but it is not expected for Li with its three electrons. 
Figure 2. Simulated diffraction patterns (left) and constructed Brillouin-Jones zones (right) (a) for $\mathrm{Si}-o C 16$; and (b) for Cs- $o C 16$ with the data from Table 2. Indices hkl for reflections selected for $\mathrm{BZ}$ constructions are indicated on the patterns. The position of $2 \mathrm{k}_{\mathrm{F}}$ is shown by dotted (red) line. See discussion in the text.
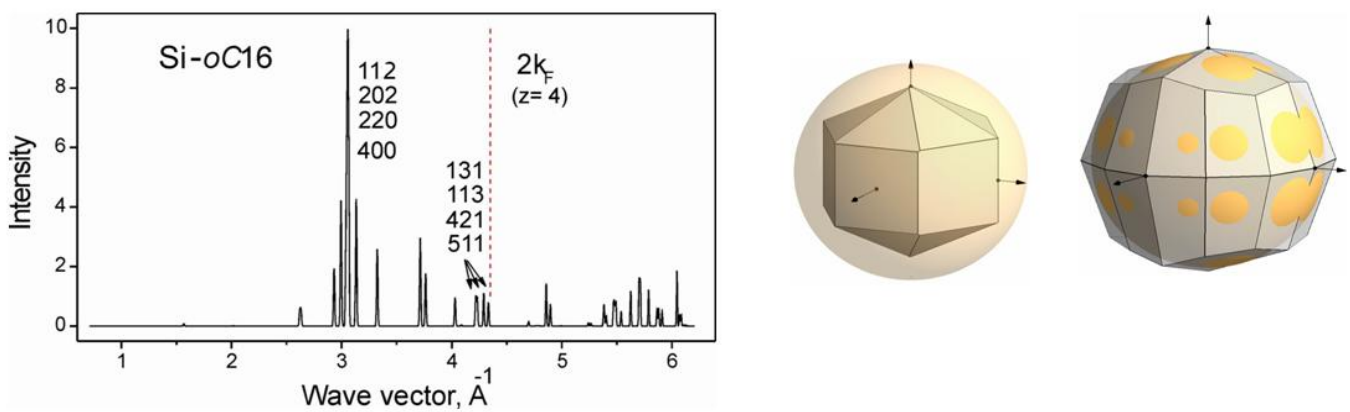

(a)
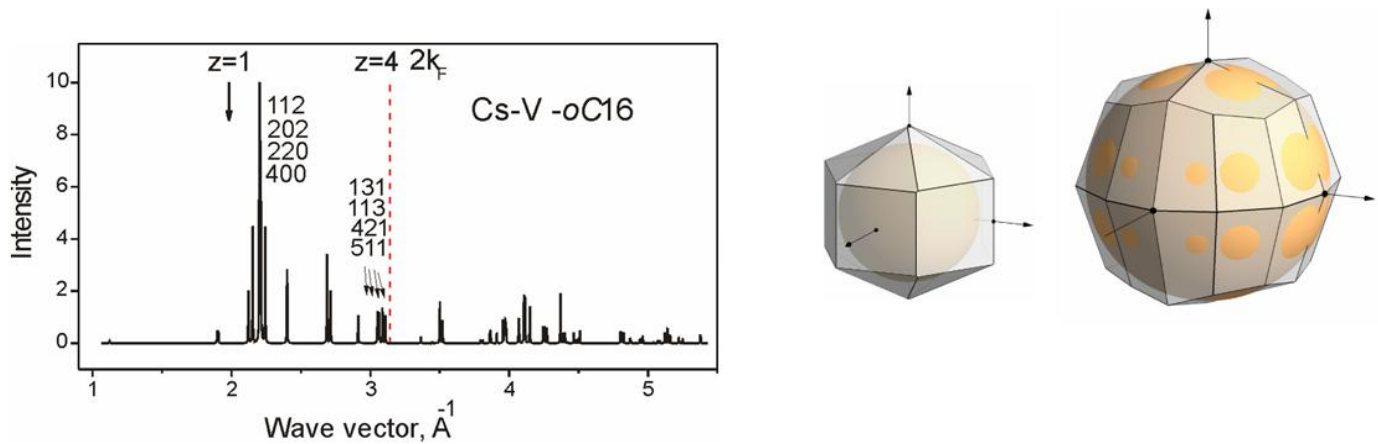

(b)

\subsection{Structure Stability and Correlation with Properties within the Hume-Rothery Mechanism}

Stability of Hume-Rothery phases in copper-based alloys, and other binary systems of noble-metals, and $s p$ polyvalent metals, have been explained by the mechanism of Brillouin zone-Fermi-sphere interactions [7,8]. Hume-Rothery effects are found to become apparent for a wide variety of material/physical objects, such as for structurally complex alloy phases, quasicrystals and their approximants [28,29]. Liquid and amorphous metals may be ascertained to obey the Hume-Rothery rules [30,31]. Connecting chemical and physical viewpoints on Hume-Rothery electron-counting rules are considered in [32], on the classic example of gamma-brasses structure.

Fermi-sphere-Brillouin zone interactions should increase under compression, providing minimization of electron band energy, which depends on volume as $\mathrm{V}^{-2 / 3}$, whereas the electrostatic energy depends as $\mathrm{V}^{-1 / 3}$ (see discussion in [2]). The $o$ C16-Cmca structure considered in this paper satisfies the criteria of Hume-Rothery mechanism for the elements $\mathrm{Si}$ and Ge with four valence electrons and there is no satisfaction of the Hume-Rothery mechanism for alkali metals, if considered monovalent. If the $o C 16$ structure in the alkali metals is considered to be stabilized by the Hume-Rothery mechanism, it is essential to assume the increase in the valence electron count to $z=4$ in these metals due to the valence band overlap with the outer core electrons.

From another point of view, atomic interactions can be analyzed using the electron localization function (ELF), which describes chemical bonding in real space [33,34]. The result of this approach is that a large amount of valence charge in the open-packed structures resides within the interstitial regions 
corresponding to the formation of "electrides", in which the interstitial electrons form the anions. Thus, an elemental phase under pressure is considered as "pseudobinary ionic compound" [35,36]. These papers report the observation of the hexagonal phases of NiAs- $h P 4$ type in $\mathrm{Na}$ and $\mathrm{K}$ (above $180 \mathrm{GPa}$ and $35 \mathrm{GPa}$, respectively), the type that is widely occurring in binary phases (see by Pearson [12]). The formation of this phase, formed in a binary system of $s p$-metals, can be well explained using the Hume-Rothery mechanism with an appropriate number of valence electrons. There are a great variety of complex phases based on $h P 4$ formed by distortions, vacancies, and superlattices to satisfy Hume-Rothery effects [37]. These considerations can help in the understanding of structural variety observed in alkali elements under different pressure-temperature conditions.

Structural properties of materials in many instances correlate with their physical properties. Fermi-sphere-Brillouin zone interactions, and degree of filling of the Brillouin zone by electron states, considerably define the properties of material like electrical resistivity, optical reflectivity, superconductivity, and others. With a high degree of BZ filling the free Fermi surface area becomes small, which is accompanied by an increase in density of state below the $\mathrm{k}_{\mathrm{F}}$. The consequences of this FS-BZ configuration are the decrease in reflectivity, increase in resistivity, appearance, and increase in superconductivity, as discussed by Hirsch [38,39].

For the phases with the oC16-Cmca structure in $\mathrm{Cs}$ and $\mathrm{Si}$, experiments show a relatively high electrical resistance and an increase in $\mathrm{T}_{\mathrm{c}}$ of superconducting state (see discussion in [2]). Electrical resistance measurements under pressure available for $\mathrm{Cs}$ found a sharp increase at the transition $t I 4-o C 16$ near $\sim 13 \mathrm{GPa}$. For Cs superconductivity emerges in $t I 4$ at $\sim 11 \mathrm{GPa}$ and riches $\mathrm{T}_{\mathrm{c}} \sim 1.3-1.5 \mathrm{~K}$ in $o C 16$ at pressure $12-14 \mathrm{GPa}$ [40]. For Si, superconducting state was observed at the transition to the metallic state with $\beta$-Sn ( $t I 4)$, and in the subsequent $o I 4$ and $h P 1$ phases with $\mathrm{T}_{\mathrm{c}} \sim 7-8 \mathrm{~K}$ at pressures $12-20 \mathrm{GPa}$, with a decrease of $\mathrm{T}_{\mathrm{c}}$ with pressure within the existence of the simple hexagonal $(h P 1)$ phase. It is noticeable that after reaching the minimum of $3.3 \mathrm{~K}$ at $37 \mathrm{GPa}$, $\mathrm{T}_{\mathrm{c}}$ starts to increase again and reaches $4.9 \mathrm{~K}$ at $40 \mathrm{GPa}$ in the $o C 16$ phase, followed by a drop in the $h c p$ phase [41]. The observed, relatively high $\mathrm{T}_{\mathrm{c}}$ values in the $o C 16$ phases can be attributed to the higher degree of filling of the Brillouin-Jones zone by the electronic states.

The drop of resistance for $\mathrm{Bi}$ at the transition of the ambient pressure semimetallic phase to $\mathrm{Bi}-\mathrm{II}-m C 4$ at $2.5 \mathrm{GPa}$ is well known from its magnitude, sharpness, and reversibility, and makes it an ideal pressure calibration point [25]. Further transition to Bi-III (host-guest) at $2.7 \mathrm{GPa}$ leads to an increase of resistance, and the high temperature phase $\mathrm{Bi}-\mathrm{IV}-o C 16$ has a resistance that is a little lower than for Bi-III. At higher pressures, the transition IV-V is accompanied by a moderately sharp drop in resistance. This behavior of resistance in $\mathrm{Bi}$ can be related now to established crystal structures of high-pressure phases. Relatively high resistance of Bi-IV is in agreement with our considerations of FS-BZ model for $o C 16$ structure. In addition, the relatively high $\mathrm{T}_{\mathrm{c}}$ values were observed for quenched $o C 16$ phases in $\mathrm{Bi}_{80} \mathrm{~Pb}_{20}$ and $\mathrm{Bi}_{70} \mathrm{In}_{30}$ alloys, $8.7 \mathrm{~K}$ and $7.9 \mathrm{~K}$, respectively [27].

In the Bi-Sn alloys of the composition $40 / 60$ at $\%$, examined in situ under pressure, the initial phase mixture $\mathrm{Bi}+\mathrm{Sn}$ was transformed to the intermediate phase after annealing the sample at $2.9 \mathrm{GPa}$ and $150{ }^{\circ} \mathrm{C}$ for one hour. The diffraction pattern observed after annealing corresponded to the $o C 16$ phase. The superconducting transition temperature $\left(\mathrm{T}_{\mathrm{c}}\right)$ in $\mathrm{BiSn}$ alloy at ambient pressure is $3.7 \mathrm{~K}$, a characteristic of $\beta-\mathrm{Sn}$ in the phase mixture with nonsuperconducting Bi. Under pressure, after the 
formation of the intermediate phase on the annealing at $363 \mathrm{~K}$ and $3.0 \mathrm{GPa}$, an increase in $\mathrm{T}_{\mathrm{c}}$ to $7.4 \mathrm{~K}$ was observed (see Reference [20] and references therein).

On the whole, in all three Bi-based systems, intermediate phases formed under pressure-temperature conditions from two-phase mixtures have the same $o C 16$ structure, identical to high-pressure high temperature form of $\mathrm{Bi}$, and these phases can be considered as extended solid solutions based on $\mathrm{Bi}-o C 16$. In addition, an atomic ordering within $8 \mathrm{f}$ and $8 \mathrm{~d}$ positions was observed for the nearly equiatomic BiSn-oC16 phase. Structural resemblance of Bi-based phases is supplemented by similar superconducting properties that have its source from the discussed Hume-Rothery mechanism.

\section{Method of Analysis}

The underlying concept of structural stability in the nearly-free electron model is based on matching of the Fermi sphere (FS) radius, $\mathrm{k}_{\mathrm{F}}$, and the distance to the Brillouin zone plane, $1 / 2 q_{h k l}$, that is half of the reciprocal vector $q_{h k l}, \mathrm{k}_{\mathrm{F}} \approx 1 / 2 q_{h k l}[7,8]$. This rule defines boundaries of phase stability by average number of valence electrons per atom for classical Hume-Rothery phases, such as fcc, bcc, and hcp. For complex phases such as $\gamma$-brass, $\mathrm{Cu}_{5} \mathrm{Zn}_{8}-\mathrm{cI52}$, it is necessary to consider the extended zone or Brillouin-Jones zone, assigned here as BZ. For complex phases, it is important as to how well the BZ accommodates the FS, which provides the gain in band structure energy to be enough to compensate the loss in electrostatic energy that arises from the formation of superlattices, vacancies, and distortions.

To analyze complex phases within the FS-BZ interactions, the program BRIZ is used, which allows to consider high symmetry structures, cubic or hexagonal, as well as structures of low symmetry: tetragonal, orthorhombic, monoclinic, or triclinic [22,37]. As an input, the following data are used: lattice parameters, number of atoms in the cell, number of valence electrons per atom, and the hkl indices of the Brillouin planes that are selected from peaks in the diffraction pattern located near $\mathrm{k}_{\mathrm{F}}$. The output data are the $\mathrm{BZ}$ volume, the $\mathrm{FS}$ volume, $\mathrm{k}_{\mathrm{F}}$, and distances to the $\mathrm{BZ}$ planes. The program gives $\mathrm{BZ}$ construction with the inscribed FS as shown in Figure 2. This approach allows qualitative estimations of stability of structure and some physical properties of materials with this structure.

Thus, with the FS-BZ consideration for the phases in compressed alkali metals $\mathrm{Li}$ and $\mathrm{Na}$, the appearance of a complex cI16 structure after bcc and fcc is evident to be of electronic origin of Hume-Rothery type, when superlattice $2 \times 2 \times 2$ is formed due to a slight atomic shift to produce a Bragg plane close to $\mathrm{k}_{\mathrm{F}}$, at that $\mathrm{Li}$ and $\mathrm{Na}$ are monovalent $[2,11]$. When considering the next phase in Na with the $o P 8$ structure, it is essential to assume Na being divalent as for the isostructulal phase AuGa [11].

The same FS-BZ analysis can be applied to the problem of incommensurate structures, by using suitable approximants, as shown for incommensurately modulated phases of phosphorous at pressures 100-137 GPa [42]. Incommensurate host-guest phases are found in alkali metals $\mathrm{Na}, \mathrm{K}$, and $\mathrm{Rb}$, and they can be considered in relation to the Hume-Rothery mechanism with the proper approximant extracting the necessary valence electron count from the FS-BZ model, as well as Bi-III host-guest phase [43]. It is important to mention that these host-guest phases in alkali elements are inline among the other high-pressure phases that are suggested to be considered within Hume-Rothery mechanism, as the $o C 16$ phase discussed in this paper. 


\section{Conclusions}

In this paper the $o C 16-C m c a$ structure is analyzed for the purpose of understanding the formation and stability of this structure in a number of elements from both sides of the Periodic Table, as well as in some binary alloys, for instance, in $\mathrm{Bi}$ alloys with $\mathrm{In}, \mathrm{Pb}$, and $\mathrm{Sn}$. Primarily, the Cmca-oC16 structure is a common high-pressure form for polyvalent metals and alloys with the average number of valence electrons $\sim 4$, and might well be found in other alloys with the constituents from groups III-VI under pressure. Stability of this rather complex phase is related to the Hume-Rothery mechanism and gaining in the valence electron energy. Construction of Brillouin zone boundaries with the Bragg plains close to the Fermi sphere results in a highly-symmetrical polyhedron with many faces contacting the Fermi sphere, and nearly filled by electron states. This configuration meets well the Hume-Rothery mechanism criteria for phase stability, explaining qualitatively the physical properties of the matter with this structure such as resistivity and superconductivity.

Unexpected formation of the $o C 16$-Cmca structure in compressed alkali elements $\mathrm{K}$, $\mathrm{Rb}$, and $\mathrm{Cs}$ brings up the question of the valence electron level hybridization with the upper core electrons. The commonly accepted $s$ - $d$ electron transition is sufficient to explain the first few high-pressure transitions in alkali metals and on further compression it is necessary to extend the degree of electron level hybridization that may account for sequences of complex high pressure phases including discussed in this paper, the $o C 16$ phase.

\section{Acknowledgments}

The author gratefully acknowledges Olga Degtyareva for valuable discussion and comments. This work is supported by the Program of Russian Academy of Sciences "The Matter under High Energy Density".

\section{Conflict of Interest}

The author declares no conflict of interest.

\section{References}

1. McMahon, M.I.; Nelmes, R.J. High-pressure structures and phase transformations in elemental metals. Chem. Soc. Rev. 2006, 35, 943-963.

2. Degtyareva, V.F. Simple metals at high pressures: The Fermi sphere-Brillouin zone interaction model. Phys. Usp. 2006, 49, 369-388.

3. Degtyareva, O. Crystal structure of simple metals at high pressures. High Press. Res. 2010, 30, 343-371.

4. Schwarz, U.; Takemura, K.; Hanfland, M.; Syassen, K. Crystal structure of cesium-V. Phys. Rev. Lett. 1998, 81, 2711-2714.

5. Hanfland, M.; Schwarz, U.; Syassen, K.; Takemura, K. Crystal structure of the high-pressure phase silicon VI. Phys. Rev. Lett. 1999, 82, 1197-1200.

6. Schwarz, U.; Jepsen, O.; Syassen, K. Electronic structure and bonding in the Cmca phases of Si and Cs. Solid State Commun. 2000, 113, 643-648. 
7. Mott, N.F.; Jones, H. The Theory of the Properties of Metals and Alloys; Oxford University Press: London, UK, 1936.

8. Jones, H. The Theory of Brillouin Zones and Electron States in Crystals; North-Holland Publishing Company: Amsterdam, Holland, 1962.

9. Hume-Rothery, W.; Coles, B.R. Atomic Theory for Students of Metallurgy; Institute of Metals: London, UK, 1988.

10. Ross, M.; McMahan, A.K. Systematics of the $s \rightarrow d$ and $p \rightarrow d$ electronic transition at high pressure for the elements I through La. Phys. Rev. B 1982, 26, 4088-4093.

11. Degtyareva, V.F.; Degtyareva, O. Structure stability in the simple element sodium under pressure. New J. Phys. 2009, 11, 063037:1-063037:16.

12. Pearson, W.B. Crystal Chemistry and Physics of Metals and Alloys; Wiley-Interscience: New York, NY, USA, 1972.

13. Kubiak, R.; Wolcyrz, M. Refinement of crystal structures of $\mathrm{AuSn}_{4}$ and $\mathrm{PdSn}_{4}$. J. Less Common Met. 1984, 97, 265-269.

14. Kittel, C. Introduction to Solid State Physics; JohnWiley \& Sons: New York, NY, USA, 1995.

15. Shannon, R.D. Revised Effective Ionic Radii and Systematic Studies of Interatomic Distances in Halides and Chaleogenides. Acta Cryst. A 1976, 32, 751-767.

16. Takemura, K.; Schwarz, U.; Syassen, K.; Hanfland, M.; Christensen, N.; Novikov, D.; Loa, I. High-pressure Cmca and hcp phases of germanium. Phys. Rev. B 2000, 62, R10603-R10606.

17. Schwarz, U.; Syassen, K.; Grzechnik, A.; Hanfland, M. The crystal structure of rubidium-VI near 50 GPa. Solid State Commun. 1999, 112, 319-322.

18. Lundegaard, L.F.; Marqués, M.; Stinton, G.; Ackland, G.J.; Nelmes, R.J.; McMahon, M.I. Observation of the $o P 8$ crystal structure in potassium at high pressure. Phys. Rev. B 2009. 80, 020101:1-020101:4.

19. Chaimayo, W.; Lundegaard, L.F.; Loa, I.; Stinton, G.W.; Lennie, A.R.; McMahon, M.I. High-pressure, high-temperature single-crystal study of Bi-IV. High Press. Res. 2012, 32, 442-449.

20. Degtyareva, V.F.; Degtyareva, O.; Allan, D.R. Ordered Si-VI-type crystal structure in BiSn alloy under high pressure. Phys. Rev. B 2003, 67, 212105:1-212105:4.

21. Degtyareva, V.F. Crystal structure of a high-pressure phase in Bi-based alloys related to Si-VI. Phys. Rev. B 2000, 62, 9-12.

22. Degtyareva, V.F.; Smirnova, I.S. BRIZ: A vizualization program for Brillouin zone-Fermi sphere configuration. Z. Kristallogr. 2007, 222, 718-721.

23. Sato, H.; Toth, R.S. Fermi Surface of Alloys. Phys. Rev. Lett. 1962, 8, 239-241.

24. Harrison, W.A. Pseudopotentials. In the Theory of Metals; Elsevier: New York, NY, USA, 1966.

25. Bundy, F.P. Phase diagram of bismuth to $130,000 \mathrm{~kg} / \mathrm{cm}^{2}, 500^{\circ} \mathrm{C}$. Phys. Rev. 1958, 110, 314-318.

26. McMahon, M.I.; Degtyareva, O.; Nelmes, R.J. Ba-IV-type incommensurate crystal structure in group-V metals, Phys. Rev. Lett. 2000, 85, 4896-4899.

27. Ponyatovskii, E.G.; Degtyareva, V.F. Specific Features of T-C-P diagrams for binary systems of B-elements. High Press. Res. 1989, 1, 163-184.

28. Mizutani, U. Introduction to the Electron. Theory of Metals; Cambridge University Press: New York, NY, USA 2001. 
29. Mizutani, U. Hume-Rothery Rules for Structurally Complex Alloy Phases; Taylor \& Francis US: London, UK, 2010.

30. Häussler, P. Interrelations between atomic and electronic structures-Liquid and amorphous metals as model systems. Phys. Rep. 1992, 222, 65-143.

31. Stiehler, M.; Rauchhaupt, J.; Giegengack, U.; Häussler, P. On modifications of the well-known Hume-Rothery rules: Amorphous alloys as model systems. J. Non-Cryst. Solids 2007, 353, 1886-1891.

32. Berger, R.F.; Walters, P.L.; Stephen Lee, S.; Hoffmann, R. Connecting the Chemical and Physical Viewpoints of What Determines Structure: From 1-D Chains to $\gamma$-Brasses. Chem. Rev. 2011, 111, 4522-4545.

33. Von Schnering, H.G.; Nesper, R. How nature adapts chemical structures to curved surfaces. Angew. Chem. Int. Ed. Engl. 1987, 26, 1059-1080.

34. Nesper, R.; Grin, Yu. Periodic Space Partitioners (PSP) and their relations to crystal chemistry. Z. Kristallogr. 2011, 226, 692-710.

35. Ma, Y.; Eremets, M.; Oganov, A.R.; Xie,Y.; Trojan, I.; Medvedev, S.; Lyakhov, A.O.; Valle, M.; Prakapenka, V. Transparent dense sodium. Nature 2009, 458, 182-186.

36. Marqués, M.; Ackland, G.J.; Lundegaard, L.F.; Stinton, G.; Nelmes, R.J.; McMahon, M.I. Potassium under pressure: A pseudobinary ionic compound. Phys. Rev. Lett. 2009, 103, 115501:1-115501:4.

37. Degtyareva, V.F.; Afonikova, N.S. Simple metal binary phases based on the body centered cubic structure: Electronic origin of distortions and superlattices. J. Phys. Chem. Solids 2013, 74, 18-24.

38. Hirsch, J.E.; Hamlin, J.J. Why non-superconducting metallic elements become superconducting under high pressure. Phys. C Supercond. 2010, 470, S937-S939.

39. Hirsch, J.E. Materials and mechanisms of hole superconductivity. Phys. C Supercond. 2012, 472, $78-82$.

40. Wittig, J. Pressure-Induced Superconductivity in Cesium and Yttrium. Phys. Rev. Lett. 1970, 24, 812-815.

41. Erskine, D.; Yu, P.Y.; Chang, K.J.; Cohen, M.L. Superconductivity and Phase Transitions in Compressed Si to 45 GPa. Phys. Rev. Lett. 1986, 57, 2741-2744.

42. Degtyareva, V.F. Electronic origin of the incommensurate modulation in the structure of phosphorus IV. J. Phys. Conf. Ser. 2010, 226, 012019:1-012019:5.

43. Degtyareva, V.F.; Degtyareva, O. Potassium under pressure: Electronic origin of complex structures. Presented at the XXVIII International Conference on Interaction of Intense Energy Fluxes with Matter, Elbrus, Kabardino-Balkaria, Russia, 1-6 March 2013.

(C) 2013 by the authors; licensee MDPI, Basel, Switzerland. This article is an open access article distributed under the terms and conditions of the Creative Commons Attribution license (http://creativecommons.org/licenses/by/3.0/). 